\begin{tabular}{|c|c|}
\hline \multicolumn{2}{c}{ Access this article online } \\
\hline
\end{tabular}

\title{
Ophthalmic emergencies in patients under 15 years of age - a single-center analysis
}

\author{
Karolina Mróz, Aleksandra Borys, Anna Chmielarz-Czarnocińska, Anna Gotz-Więckowska \\ Department of Ophthalmology, Poznan University of Medical Sciences, Poznan, Poland
}

\begin{abstract}
Aim of the study: To analyze the reasons for referrals of patients under the age of 15 to the Ophthalmic Emergency Department (ED), to determine the type of services provided and to define the age range of children presenting to the ED most frequently.

Material and methods: The retrospective review included $537 \mathrm{pa}-$ tients below 15 years old who presented to the ED of the Ophthalmology Department of Poznan University of Medical Sciences between November 2017 and February 2019.

Results: 250 girls and 287 boys aged from 10 days to 15 years old presented to the ED. Children less than 1 year old constituted $13.4 \%$ of patients, patients between 1 and 3 years old constituted $21.8 \%$, and those between 4 and 7 constituted $28.7 \%$. The largest group consisted of patients between 8 and 15 years old (36.1\%). Injuries constituted 71 of referrals (13.2\%). The patients most
\end{abstract}

frequently complained of redness of the eye, discharge and eye pain. The most frequently diagnosed condition was conjunctivitis (218 patients, $40.6 \%$ ), and the second most common was stye or chalazion (51 pa-tients, $9.5 \%)$. Twenty-six patients $(4.8 \%)$ required hospitalization, 4 in the ophthalmic ward $(0.7 \%)$. Two hundred seventy patients $(50.2 \%)$ were advised to visit an ophthalmologist for a check-up or to have a planned refraction examination.

Conclusions: Patients under 15 years old reported to the ophthalmic ED most commonly for non-traumatic reasons. The dominant diagnosis was conjunctivitis. The most common recommendation was mandatory control in the outpatient ophthalmic clinic. The most numerous group among the patients was that of children aged 8 to 15 years.

KEY WORDS: pediatric ophthalmology, ophthalmic emergency department, eye injuries, conjunctivitis.

\section{INTRODUCTION}

Ophthalmic emergencies represent approximately 1-6\% of presentations to the general emergency room worldwide [1] and this number is expected to increase [2]. Such conditions, especially untreated, may result in severe problems with the visual system, including even total blindness [3]. In some cases, only a prompt diagnosis and treatment may prevent severe complications of initially harmless diseases. Cooperation with children may be sometimes challenging, making examination more problematic than in the adult population.

At the Department of Ophthalmology, Poznan University of Medical Sciences, the Ophthalmic Emergency Department (ED) was established and started admitting patients - adults as well as children - in November 2017. As ocular emergencies in children are an underinvestigated research topic, this study was conducted to examine the causes of referrals of this group of patients to the ED and to analyze the recommendations given to them.
The aims of this study were:

1. To analyze the reasons for referrals of patients under the age of 15 to the Ophthalmic ED.

2. To determine the type of services provided.

3. To define the age range of children visiting the Ophthalmic ED most frequently.

4. To specify whether the Ophthalmic ED for children should be a part of the pediatric or eye department.

\section{MATERIAL AND METHODS}

In a cross-sectional retrospective study, detailed information about patients under the age of 15 who reported to the Ophthalmic ED between November 2017 and February 2019 was recorded. Demographic data including age, sex, reasons for patients' referral, diagnosis, administered drugs and other recommendation as well as the information whether hospitalization was required were collected in a specially designed form. 


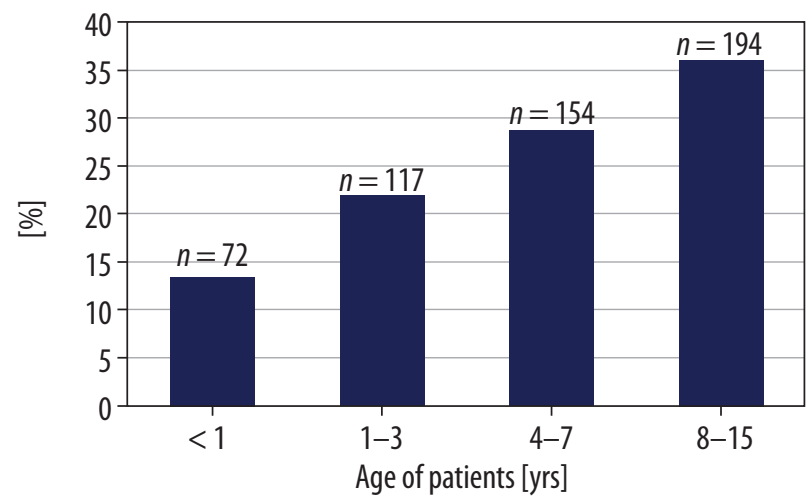

Figure 1. Age structure of admitted patients

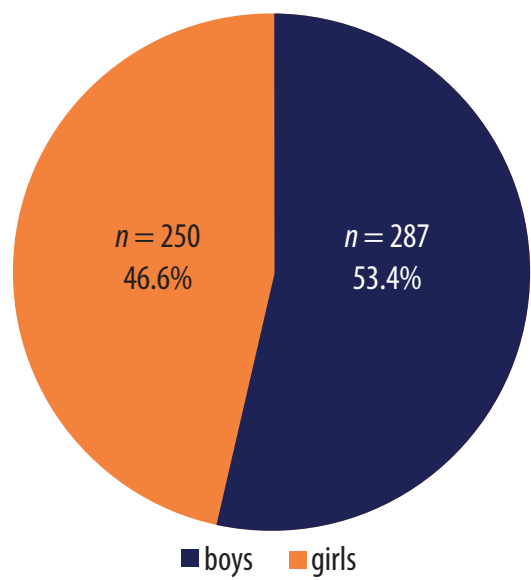

Figure 2. The sex structure admitted patients

\section{RESULTS}

Five hundred thirty-seven patients aged between 10 days to 15 years old were included in the study. The average age of the admitted patients was 6 years. The most numerous group was that of patients between 8 and 15 years of age (36.1\%). The least numerous group was that of children under the age of 1 year (13.4\%). Children aged between 1 and 3 years con- stituted $21.8 \%$ (Figure 1). Two hundred fifty (46.6\%) of them were girls, and 287 (53.5\%) were boys (Figure 2).

Four hundred sixty-six patients $(86.8 \%)$ visited the eye emergency department due to non-traumatic reasons and 71 patients (13.2\%) reported with an injury. The most common complaint was redness of an eye. This symptom was reported by 217 (40.4\%) patients (Figure 3).

Conjunctivitis was the most frequently diagnosed disease (in 218 patients, $41.0 \%$ ). The next most common conditions were: sty and chalazion - 51 patients $(9.5 \%)$, presence of a foreign body -46 patients $(8.6 \%)$ and injury of the eye -46 patients (8.6\%) (Figure 4$)$.

Four hundred seventy patients, $87.5 \%$ of all admitted patients, were prescribed one or more than one drug. Three hundred seventy-two patients $(69.3 \%)$ were given topical antibiotics. The second most frequently recommended group of medications was that of moisturizing drops, which were prescribed to 134 patients (25.0\%). All groups of drugs prescribed are listed in Figure 5.

Patients were also given other recommendations. The single most common (270 patients, 50.2\%) advice was to schedule an ophthalmologic visit or a check-up. Twenty-six patients $(4.8 \%)$ required hospitalization, $4(0.7 \%)$ of them in the ophthalmic ward to undergo surgical procedures. Forty patients $(7.5 \%)$ were referred to another specialist, most often to the neurologist (18 patients, 3.3\%) (Figure 6).

\section{DISCUSSION}

Proper organization of the ophthalmic emergency care dedicated to children is an important challenge. According to the statistics worldwide, each year 160,000-280,000 children under the age of 15 suffer from eye injuries that require hospitalization [4]. Thirty-five percent of severe ocular trauma happens to children, mostly under the age of 12 [5]. The recognized risk factors which predispose to eye trauma are age below 5 years old, male gender and lack of supervision. Usu-

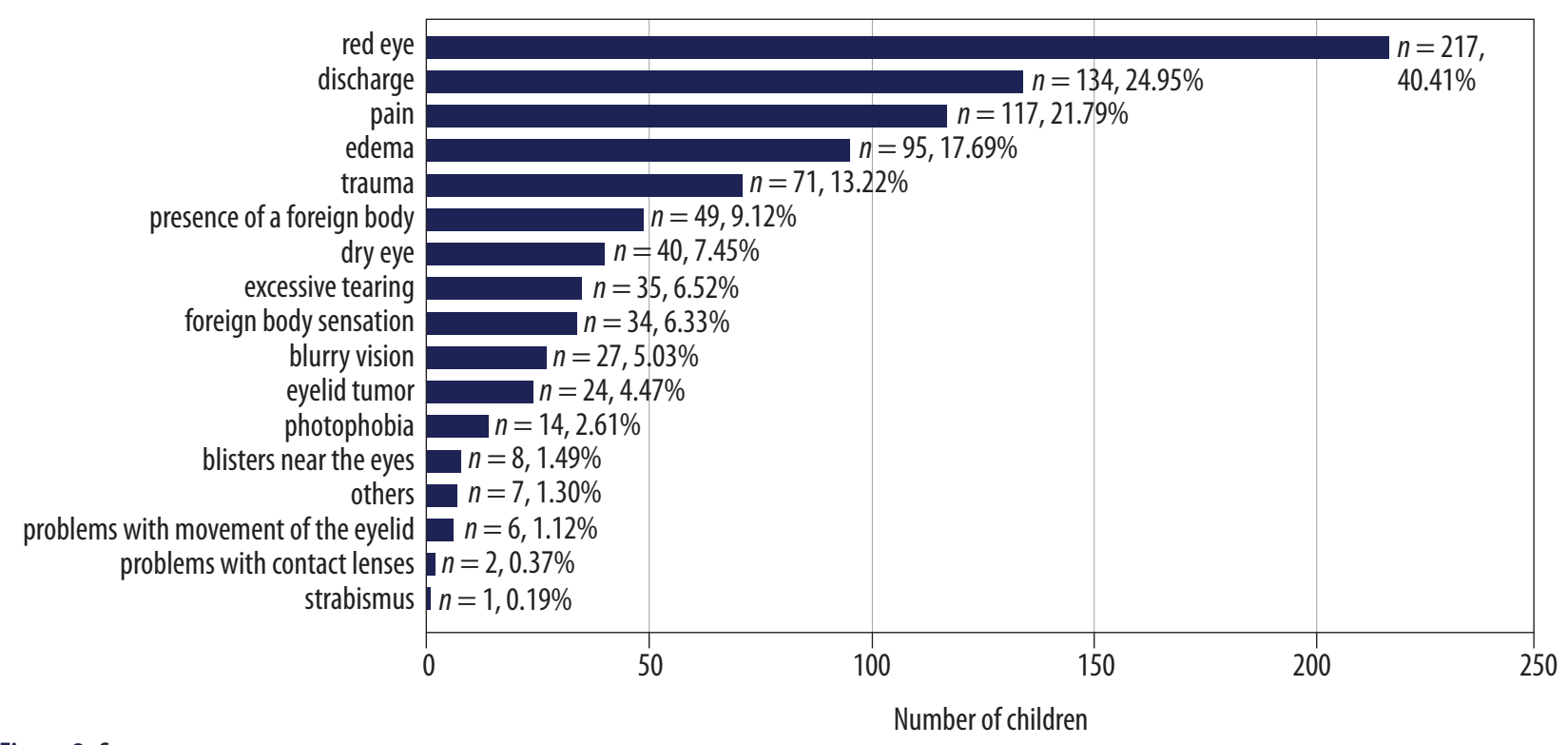

Figure 3. Symptoms 


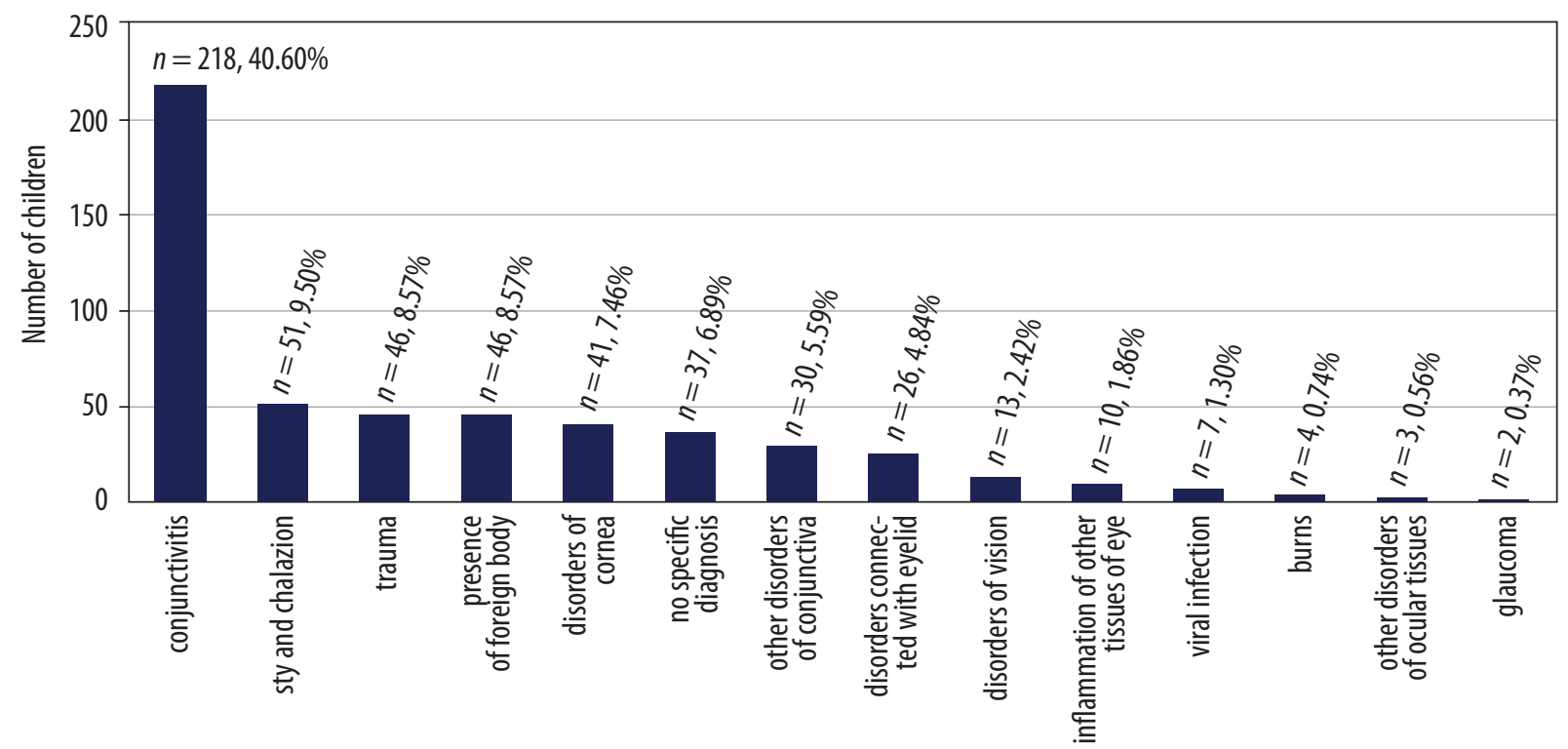

Figure 4. Diagnosis

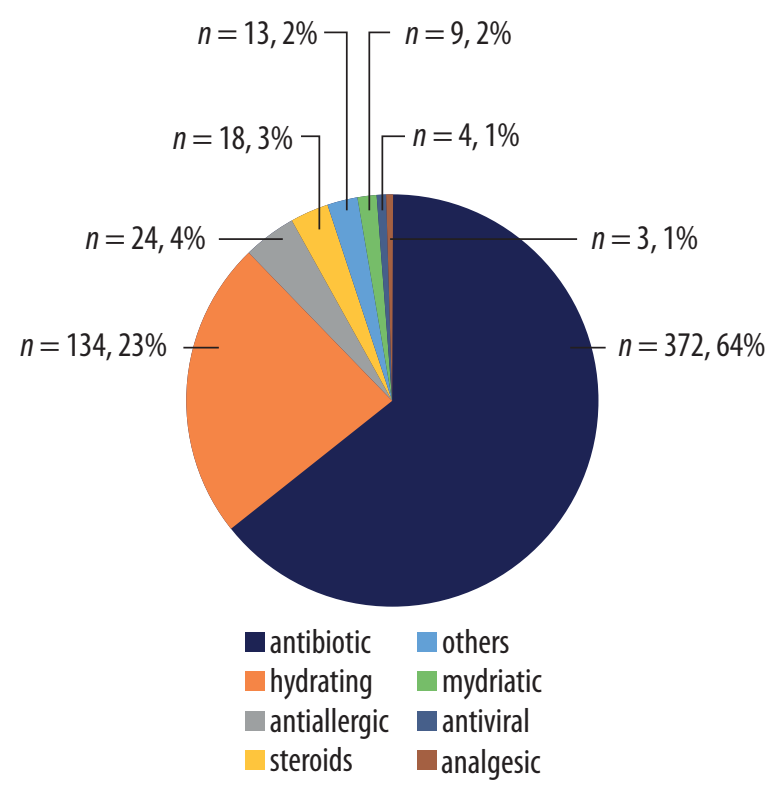

Figure 5. Prescribed drugs

ally, these are preventable events [6]. Traumas are the most common cause of children's unilateral blindness $[7,8]$.

Among our patients, injuries accounted for only $13.2 \%$ of the reasons for the Ophthalmic ED presentations. In a similar survey conducted in the United States [9], eye injuries were the reason for $36.6 \%$ of all visits, whereas in the group of pediatric patients aged 5 years old or less the figure was $25.0 \%$ and in the group of 13-18 year-olds it was $40.4 \%$. In Singapore [10], Karachi (Pakistan) [11], Pehawar (Pakistan) [12] and France [13], 53.5\%, 39.7\%, $47.16 \%$ and $45.2 \%$, respectively, of the patients were admitted to the ED due to an eye injury. Similar results were obtained in a study conducted in Korea. The incidence of ocular traumas in this research was the highest between 20 and 29 years of age and less common in the groups of 0-10 and 10-20 year-olds [13].

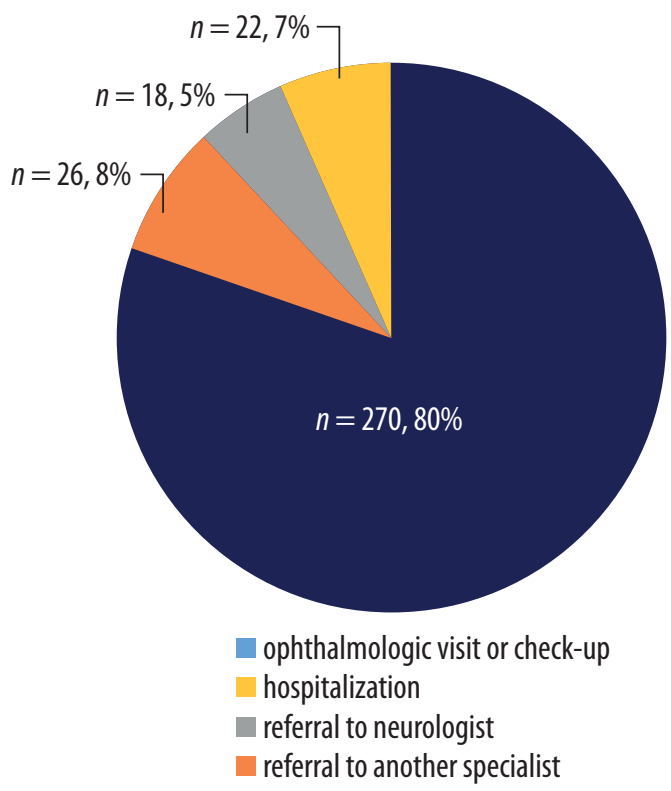

Figure 6. Recommendations given

More than half of the analyzed visits were due to conjunctivitis, styes or chalazions - common conditions, which do not pose a threat to vision. Other studies show that also in adults who reported to the ED conjunctivitis was the most frequently diagnosed disease $[3,9,14,15]$. A study conducted in Romania revealed that in infancy the most common cause of presentations to the ED was inflammatory diseases, which were also most frequently diagnosed in children. Conjunctivitis was nearly as common as injuries to the eye, whereas traumas had their peak in adulthood [14]. It is considered that injuries are the most common reason for reports to the ED in developed countries, while eye infections are dominant in developing countries [15]. It is important to stress that conjunctivitis can be successfully treated in ophthalmology outpatient clinics or by general practitioners [16]. 
Nearly $50 \%$ of visits to the eye emergency department were due to non-urgent causes and were probably unnecessary. Similar outcomes were obtained in the United States [9] and Karachi (Pakistan) [11]. The research conducted in the United States revealed that $44.3 \%$ of visits were non-urgent, only $41.2 \%$ were categorized as urgent, and $14.5 \%$ were left uncategorized. In this study, the most common causes of urgent visits were: corneal abrasion, corneal foreign body and contusion of eye and orbital tissues. The primary cause of non-urgent visits was, as in our study, conjunctivitis [9]. Such ratio of urgent to unurgent visits might be the result of the low availability of general practitioners, not only in Poland, but also in other countries.

Some of the patients (6.9\%) left the Ophthalmic ED without any specific diagnosis. Most of them did not require immediate treatment. The vast majority $(95.2 \%)$ of patients were discharged following the initial consultation. The remaining patients were admitted to the ophthalmic ward or transferred to other departments if their symptoms indicated systemic diseases. $4.8 \%$ of patients required hospitalization. In research conducted in the United States [9] about $10 \%$ of patients who presented to the ED were hospitalized, but nearly $30 \%$ of them were over 65 years of age, while they constituted only $7.8 \%$ of the analyzed population.

Only $4.8 \%$ of patients from the study group required hospitalization and even a lower number of cases required surgical treatment $(0.7 \%)$. Similar outcomes were obtained in a study carried out in Paris, where $3.02 \%$ of patients who presented to the ophthalmic ED were hospitalized [13].

Considering these small numbers, a joint Ophthalmic ED for adults and children seems to be an accurate solution. A medical facility on call must provide conditions for general anesthesia, surgical procedures and hospitalization. The optimal solution seems to be an ophthalmology ward for adults with a unit for children.

\section{CONCLUSIONS}

It seems that the location of the pediatric eye emergency department at the ophthalmology ward for adults, after meeting the necessary requirements, is an appropriate solution. Patients under 15 years of age reported to the Ophthalmic ED most often for non-traumatic reasons. The most frequent diagnosis was conjunctivitis. Patients usually received outpatient counselling, while four of them required surgical treatment. The most common recommendation was mandatory control in the outpatient ophthalmic clinic. The most numerous group of patients was that of children aged 8 to 15 years.

\section{DISCLOSURE}

The authors declare no conflict of interest.

\section{References}

1. Jafari A, Bozorgui S, Shahverdi N, et al. Different causes of referral to ophthalmology emergency room. J Emerg Trauma Shock 2012; 5: $16-22$.

2. Smith H, Daniel C, Verma S. Eye casualty services in London. Eye 2013; 27: 320-328.

3. Scruggs D, Scruggs R, Stukenborg G, et al. Ocular injuries in trauma patients: an analysis of 28,340 trauma admissions in the 2003-2007 National Trauma Data Bank National Sample Program. J Trauma Acute Care Surg 2012; 73: 1308-1312.

4. Vaziri K, Schwartz SG, Flynn HW, et al. Eye-related Emergency Department visits in the United States, 2010. Ophthalmology 2016; 123: 917-919.

5. Abbott J, Shah P. The epidemiology and etiology of pediatric ocular trauma. Surv Ophthalmol 2013; 58: 476-485.

6. LaRoche G, McIntyre L, Schertzer R. Epidemiology of severe eye injuries in childhood. Ophthalmology 1988; 95: 1603-1607.

7. Good W, Carden S. Accidental trauma in children in Taylor and Hoyt's Pediatric Ophthalmology and Strabismus. $5^{\text {th }}$ ed. Elsevier, Edinburgh 2017; 715-724.

8. Gotz-Więckowska A, Pawlak M. Praktyczna okulistyka dziecięca. Górnicki Wydawnictwo Medyczne, Wrocław 2020.

9. Channa R, Zafar SN, Canner JK, et al. Epidemiology of eye-related Emergency Department visits. JAMA Ophthalmol 2016; 134: 312-319.

10. Voon LW, See J, Wong TY. The epidemiology of ocular trauma in Singapore: perspective from the emergency service of a large tertiary hospital. Eye (Lond) 2001; 15:75-81.

11. Baig R, Ahmad K, Zafar S, et al. Frequency of ocular emergencies in a tertiary care setting in Karachi, Pakistan - It is time to reduce unnecessary visits. J Pak Med Assoc 2018; 68: 1493-1495.

12. Jan S, Khan S, Khan M, et al. Ocular emergencies. J Coll Physicians Surg Pak 2004; 14: 333-336

13. Mayouego Kouam J, Epée E, Azria S, et al. Epidemiological, clinical and therapeutic features of pediatric ocular injuries in an eye emergency unit in Île-de-France. J Fr Ophtalmol 2015; 38: 743-751.

14. Boo S, Jong S, Young S. Clinical features of ocular trauma in Emergency Department. Korean J Ophtalmol 2004; 18: 70-78.

15. Ovidiu S, Simfora 0, Lăcrămioara S. Epidemiology of ocular emergencies in Cluj ophthalmology clinic. Rom J Ophthalmol 2016; 60: 165-169.

16. Sánchez T, Galindo F, Iglesias C, et al. Epidemiologic study of ocular emergencies in a general hospital. Arch Soc Esp Oftalmol 2004; 79: 425-431.

17. Girard B, Bourcier F, Agdabede I, Laroche L. Activity and epidemiology in an ophthalmological emergency center. J Fr Ophtalmol 2002; 25: 701-711. 